\title{
Prácticas empleadas por fumigadores de plaguicidas del medio y bajo Sinú departamento de Córdoba
}

\author{
Practices used by pesticides fumigators from \\ the middle and low Sinú department of Córdoba
}

\author{
Concepción E. Amador ${ }^{*}$, José M. Luna², Elsy C. Puello³ \\ Recibido para publicación: Septiembre 6 de 2016 - Aceptado para publicación: Abril 18 de 2017
}

\begin{abstract}
RESUMEN
En el sector agrícola, de la mayoría de los países, existe un uso masivo de plaguicidas, y un déficit de conocimiento de los usuarios sobre el manejo y riesgos de éstos, siendo causa de intoxicaciones de tipo laboral. El objetivo de esta investigación fue determinar las prácticas y conocimientos sobre manejo de agroquímicos en los fumigadores de cultivos modales del Medio y Bajo Sinú del departamento de Córdoba, Colombia. Se realizó un estudio descriptivo, con abordaje cuantitativo mediante muestreo no probabilístico, entrevistando a 486 fumigadores. Entre los resultados se destaca que $89,8 \%$ carece de conocimiento válido sobre riesgos producidos por los químicos utilizados y no utilizan las medidas de protección. El 89\% transporta agroquímicos con otros insumos y alimentos, $87 \%$, desecha los envases a las orillas de los cultivos; $14,4 \%$ realiza habitualmente prácticas de manejo seguras; $13,1 \%$ indicó que reutilizan los recipientes de plaguicidas para transportar líquidos para su consumo. Entre los compuestos químicos más utilizados, 23,7\% corresponde al grupo de los órgano fosforados con aparente predilección por el uso del herbicida glifosato; $43,8 \%$ de los fumigadores reportaron el uso de compuestos de toxicidad III y $25,9 \%$ de toxicidad IV; $20,8 \%$ de los organoclorados utilizados son de uso prohibido en Colombia tales como Aldrín, Tiodan, Lindano y DDT. Se concluye que el uso de los plaguicidas entre los fumigadores del Medio y Bajo Sinú se desarrolla en condiciones de desconocimiento e ingenuidad sobre manejo seguro de plaguicidas siendo un riesgo potencial a la salud humana y ambiental.
\end{abstract}

Palabras clave: Plaguicidas, exposición laboral, experiencias, conocimiento.

\begin{abstract}
In the agricultural sector, in most countries, there is a massive use of pesticides, and a deficit of knowledge of users on the management and risks of them, which can be a cause of labor type of poisoning. The objective of this research was to determine the practices and knowledge on management of agrochemicals in the crop dusters manners of the Middle and Lower Sinú in Cordoba (Colombia). A descriptive study, with quantitative approach using nonprobability sampling, interviewing 486 sprayers was carried out. Among the results, it was observed that $89.8 \%$ of the sample did not have knowledge about the risks produced by the chemicals used and how to protect themselves. A $89 \%$ of them transport agrochemicals with other inputs and food, $87 \%$, discard the packaging on the shores of crops; $14.4 \%$ usually safe management practices; $13.1 \%$ indicated that reuse pesticide containers for transporting liquids for consumption. Among the most commonly used chemical compounds, $23.7 \%$ corresponded to the group of component fertilizers with apparent predilection for the use of the herbicide glyphosate; $43.8 \%$ of the Exterminators reported the use of toxic compounds III and $25.9 \%$ of toxicity IV; $20.8 \%$ of the organochlorides used are prohibited in Colombia such as Aldrin, DDT, lindane, and Tiodan. It was concluded that the use of pesticides among the exterminators of the Middle and Lower Sinú is due to lack of knowledge and naivety regarding safe handling of pesticides, which is a potential risk to human and environmental health.
\end{abstract}

Keywords: Pesticides, occupational exposure, experience, knowledge.

\footnotetext{
${ }^{1 *}$ M. Sc. Docente Titular Departamento de Enfermería, Universidad de Córdoba, Montería - Colombia. Email: conceamador@yahoo.es Dirección Carrera $6^{\mathrm{a}} \mathrm{N}^{\mathrm{o}}$ 76-103. Montería Córdoba. Colombia.

${ }^{2}$ M. Sc. Docente Titular. Departamento de Ingeniería Agronómica y Desarrollo Rural, Universidad de Córdoba, Montería - Colombia. Email: moises1951luna@gmail.com Dirección: Carrera 6 ${ }^{a}$ № 76-103. Montería Córdoba. Colombia.

${ }^{3}$ M. Sc. Docente Asociada. Departamento de Enfermería, Universidad de Córdoba, Montería - Colombia. Email: epuello@correo unicordoba.edu.co Dirección Carrera 6 № $^{\mathrm{a}}$ 76-103. Montería Córdoba. Colombia.
} 


\section{INTRODUCCIÓN}

Para el presente estudio los investigadores conceptuaron "cultivo modal" como aquella especie vegetal que es producida de forma tradicional y/o empresarial intensiva en una región y/o agroecosistema por presentar amplia adaptación a las condiciones de oferta ambiental y además sostiene una significativa demanda en los mercados locales, regionales, nacionales y pudiendo aún trascender las fronteras nacionales.

Para el abastecimiento de alimento de la población mundial, 90\% depende de 15 tipos de cultivos vegetales y siete especies de animales. A pesar de todos los esfuerzos realizados, las plagas destruyen anualmente cerca del $35 \%$ de las cosechas en todo el mundo (Badii et al. 2005) y para controlarlas o eliminarlas inevitablemente se incrementa el uso de agroquímicos. En el sector agrícola de la mayoría de los países existe un uso masivo de plaguicidas; esta amplia utilización aunada al escaso conocimiento de los usuarios sobre los riesgos que el empleo de estos produce, facilita la aparición de intoxicaciones de tipo laboral, accidental o intencional. En este orden, (Ortiz et al. 2011), señalan que el uso de los agroquímicos por parte de las comunidades rurales se realiza sin normas claras de bioseguridad, condición cultural que genera diversos casos con altos niveles de contaminación por el manejo inadecuado de productos químicos. En forma general, los trabajadores agrícolas afrontan riesgos muy altos para su salud y su vida, pero de manera específica los más expuestos son los fumigadores de cultivos, así mismo, también se arriesgan todas aquellas personas que por su actividad laboral emplean plaguicidas, herbicidas y fertilizantes entre otros compuestos agroquímicos.

El estado del arte de la literatura revisada permite deducir que los sistemas de evaluación de los riesgos de toxicidad se han caracterizado por orientar el problema en dirección hacia la eficacia y eficiencia en la protección de los diferentes tipos de plantas o de las plagas que se desean eliminar ó controlar. Los adelantos científicos en la investigación de las consecuencias de intoxicaciones por dichos compuestos comienzan a brindar un nivel de información hasta hace poco inconcebible, sobre todo respecto a la capacidad de evidenciar la exposición (Kaczewer 2009).

Ortiz et al. (2011) citan el concepto de plaguicida de la Organización de las Naciones Unidas para la Alimentación y la Agricultura, entendido como "una sustancia o mezcla de sustancias destinadas a prevenir, destruir o controlar cualquier plaga, incluyendo vectores de enfermedad humana o animal, especies indeseadas de plantas o animales capaces de causar daños o interferir de cualquier otra forma con la producción, procesamiento, almacenamiento, transporte o mercado de los alimentos, otros productos agrícolas, madera y sus derivados o alimentos animales, o que pueden ser administrados a los animales para el control de insectos, arácnidos u otras plagas en sus organismos" y aunque son innegables los beneficios que han aportado los plaguicidas a la civilización tanto en el campo de la salud pública en lo relacionado con el control de vectores de enfermedad, como en la agricultura en el control de plagas para mejoramiento de la productividad (Ramírez y Lacasaña 2001). Es necesario reconocer e informar los daños causados por el uso indiscriminado, excesivo ó poco cauteloso de estos productos.

Es reconocido que las sustancias químicas que conceden los principios activos a los plaguicidas también generan algunas propiedades tóxicas en los humanos por ejemplo el glifosato tiene un efecto citotóxico y genotóxico en linfocitos humanos in vitro y expuestos durante 48 horas al tóxico (Díaz 2011) y actúan de manera negativa sobre el medio ambiente; otros agroquímicos como Aldrín, Tiodan, Lindano, 
DDT y otros organoclorados fueron prohibidos en el territorio nacional porque sus propiedades físico-químicas (ICA 2004) les conceden prolongada persistencia en el medio y su cinética de dispersión favorece la problemática de salud humana y ambiental (Badii et al. 2007; Barba-Ho 2011).

Las propiedades químico-biológicas de degradabilidad de los plaguicidas tiene una influencia negativa sobre el medio ambiente (Badii et al. 2007). Estas propiedades hacen referencia a que la actividad de un plaguicida puede ser permanente o bien puede disminuir con el tiempo en función de su descomposición, ya sea química (quimiodegradabilidad), por acción de la luz (fotodegradabilidad), o por acción de sistemas microbianos (biodegradabilidad). En el mismo sentido, muchos autores han demostrado que los plaguicidas que persisten más tiempo en el ambiente tienen una mayor probabilidad de interacción con otros elementos del sistema. Por otro lado, si su vida media y su persistencia es mayor a la frecuencia con la que se aplica, el plaguicida tiende a acumularse tanto en los suelos como en la biota; estas condiciones han conducido a que los plaguicidas de más alta persistencia tengan prohibido su uso en el territorio nacional colombiano según regulaciones del Ministerio de Agricultura en la Resolución 447 de 1974 y en la Resolución 1311 de 2001 del Instituto Colombiano Agropecuario (ICA).

Por su vida media, los plaguicidas se clasifican en permanentes, persistentes, moderadamente persistentes y no persistentes (Barba-Ho y Becerra 2011). Todos los plaguicidas químicos son tóxicos y pueden producir enfermedades agudas, si ingresa al organismo una dosis excesiva por contacto inhalación o ingestión, de igual forma, los plaguicidas también pueden ocasionar enfermedades crónicas cuando se absorben repetidamente pequeñas dosis (Ortiz et al. 2011).
Los agroquímicos con efecto plaguicida, generan repercusiones nocivas sobre la salud humana, por cuanto tienen la propiedad de inhibir la actividad de la enzima acetilcolinesterasa ocasionando malformaciones, alteración del sistema nervioso y en ocasiones la muerte por toxicidad aguda (Duran-nah y Colli-Quintal. 2000); además son capaces de intervenir procesos importantes del equilibrio de los ecosistemas naturales al limitar o suprimir la microflora y microfauna (Barreto 1989); estos resultados aportan información relevante, en la medida en que evidencian la vulnerabilidad y necesidad de protección en salud de los trabajadores rurales que se desempeñan ocupacionalmente como jornalerosfumigadores así como de los recursos naturales susceptibles de ser afectados por dichas sustancias químicas tóxicas.

Los cultivos modales con proyección empresarial más significativos en el departamento de Córdoba son arroz, pastos y forrajes, maíz, algodón, yuca y ñame, los cuales requieren de apoyo agroquímico para control de plagas y malezas que pueden afectar de forma negativa la producción industrial de éstos. Es de interés resaltar, que al estimar el peso de los plaguicidas con los datos de Fedesarrollo en relación con los costos de producción, se obtiene que el porcentaje de participación de los insumos plaguicidas en grandes extensiones de cultivos, indica una alta participación de insumos plaguicidas, que para el caso del maíz en Córdoba corresponde a $27.84 \%$ del costo de insumos (Fedesarrollo-Iquartil 2013).

En Colombia la exposición a plaguicidas se ha convertido en un problema de Salud Pública, en los reportes anuales disponibles al Sistema Nacional de Vigilancia en Salud Pública SIVIGILA, se notificó en 2012 un total de 27.126 y en 2013 un total anual de 27.252 casos de intoxicaciones por sustancias químicas. Este reporte indica en los años mencionados las intoxicaciones por plaguicidas se ubicaron en 
el segundo renglón de importancia $(29,17 \%)$ siendo superadas por las intoxicaciones por medicamentos (SIVIGILA 2014).

En el año 2015 se identificó que 24,36\% de los 316 casos atendidos por intoxicaciones agudas en el principal hospital de referencia de Córdoba, correspondió a toxicidad por plaguicidas. (Terán et al. 2015).

En ese sentido, un informe de la Federación de Aseguradores Colombianos (FASECOLDA 2013) señala que el número de enfermedades laborales sobre la actividad de agricultura, ganadería, caza y silvicultora encabeza la distribución de tasas de enfermedades laborales con 339,17 casos confirmados en el año 2012, equivalentes a 11\% de total de casos reportados en Colombia, ocupando el primer lugar de incidencia de enfermedades asociadas al trabajo, así mismo reporta el informe que el sector agricultura tiene cifras de accidentalidad laboral en Colombia de $13,64 \%$ en tanto que países con Chile y México tienen cifras de $6,6 \%$ y $3,29 \%$ respectivamente. Bajo estas consideraciones, se planteó la necesidad de identificar las prácticas y los conocimientos sobre manejo de agroquímicos que tienen los fumigadores de cultivos modales del Sinú Medio y Bajo del departamento de Córdoba.

\section{MATERIALES Y MÉTODOS}

Se realizó un estudio descriptivo transversal, con abordaje cuantitativo realizado en los asentamientos humanos rurales de la región geográfica del Sinú Medio y Bajo, Región Caribe en el departamento de Córdoba, Colombia, América del Sur, en los cuales se desarrollan actividades agrícolas.

Los participantes fueron seleccionados mediante muestreo no probabilístico, por conveniencia. Se entrevistaron 486 trabajadores agrícolas que ejercen principalmente el rol de fumigadores de cultivos modales de maíz y algodón. Se utilizó como fuente primaria el fumigador, (aplicador de agroquímicos) quien se localizó en las áreas rurales de los municipios de Montería, Cereté, Lorica y San Pelayo, que conforman la zona geográfica estudiada.

La recolección de datos se realizó mediante entrevistas estructuradas para evaluar las condiciones de salud y trabajo que involucraba interrogantes sobre características sociales y demográficas, tipo de plaguicidas utilizados, transporte de los plaguicidas del sitio de compra a la zona rural y de los sitios de almacenamiento al área de aplicación; lugar donde se guardan las bombas de espalda, prácticas de descarte de los recipientes y conocimientos sobre los riesgos para la salud y para el ambiente de estos compuestos. La metodología incluyó observación directa de los puestos de trabajo, realizada por especialistas en salud ocupacional en las cuales se verificó por inspección el uso de Elementos de Protección Personal (EPP), el estado de las bombas de espalda y lugar de almacenamiento. Todos los participantes tuvieron la oportunidad de decidir participar o no como sujetos de estudio previa información sobre el mismo, sus métodos y alcances.

Para las clasificaciones que dan cuenta de los objetivos, se procedió de la siguiente manera: los nombres comerciales (y/o aproximaciones de ellos) que fueron referidos por los fumigadores mediante encuesta por entrevista, posteriormente fueron categorizados según el nivel de toxicidad, grupo químico, nivel de permisividad (prohibidos, permitidos) y efecto plaguicida.

Para construir la información sobre la composición de los plaguicidas, toxicidad y nivel de legalidad del plaguicida en Colombia, se solicitó a cada operario que nombrara el compuesto que más utilizaban; debido a la diversidad de respuestas (nombres químicos, comunes, regionales o comerciales de los diferentes laboratorios,) se procedió de manera inicial a agruparnombres, comunes, coloquiales, 
genéricos y/o comerciales enunciados por los fumigadores en la entrevista, seguidamente se les agrupó por compuesto químico, efecto esperado y por nivel de toxicidad; este listado fue comparado con la Norma Técnica Colombiana NTC-1188; y con los registros ICA que permiten diferenciar el estado de legalidad de los plaguicidas en Colombia.

La información debidamente agrupada y categorizada según las variables de interés, se procesó en el programa Excel versión 6.0, los resultados se presentan utilizando las mediciones propias de la estadística descriptiva.

\section{RESULTADOS Y DISCUSIÓN}

Características sociales y demográficas. Los jornaleros-fumigadores, sujetos de estudio, trabajan de manera informal, con salario a destajo y sin ningún tipo de seguridad social; el rango de edad de estos trabajadores agrícolas fue de 16 a 75 años, con un promedio de 49,5 años; se destaca la presencia de 13 adultos mayores $(2,67 \%)$ que aún hacen parte de la fuerza laboral en esta actividad económica a pesar de la mayor susceptibilidad a los efectos nocivos de los plaguicidas debido a la mayor edad. La distribución del género de los trabajadores que manipulan plaguicidas en el campo del Medio y Bajo Sinú, permite observar que las mujeres tienen una menor participación en las labores del campo que obedece a una razón estadística de 19:1, relación que indica la presencia laboral de una mujer por cada diecinueve hombres en la misma fuente de trabajo. Porcentaje que aunque aparentemente es mínimo, reviste gran importancia en salud pública por las implicaciones y efectos adversos que tienen los tóxicos en la población femenina y en los productos gestacionales de éstas.

La distribución de la ocupación de los jornaleros-fumigadores permite identificar que $73,46 \%(n=362)$ ejerce de manera exclusiva la labor de fumigador (aplicador de agroquímicos) y $26,54 \% \quad(n=129) \quad$ realiza además otras funciones relacionadas con el manejo de plaguicidas tales como almacenamiento, distribución, compra y transporte entre otros. El porcentaje de analfabetismo encontrado entre los fumigadores encuestados corresponde a $42,80 \%$ y es considerado muy alto $(n=208)$, lo cual es importante pues esta condición disminuye ampliamente, la probabilidad que estos trabajadores tengan un manejo adecuado de los plaguicidas y del cuidado de su salud al no saber leer o comprender las indicaciones de las etiquetas.

Agroquímicos-plaguicidas utilizados. Los fumigadores refieren que utilizan un total de veinticuatro (24) compuestos agroquímicos de uso frecuente en el Medio y Bajo Sinú cordobés. En la zona estudiada, los compuestos más utilizados son los órganos fosforados con 23,7\% $(n=115) ; y$ entre ellos el herbicida glifosato bajo diversas concentraciones, que se agruparon en dicha categoría, debido a las diferentes mezclas químicas. En cuanto al nivel de toxicidad de los compuestos químicos utilizados (Tabla 1), los hallazgos muestran que los niveles de toxicidad III y IV son los más utilizados por parte de los trabajadores entrevistados; en la muestra 1,9\% $(\mathrm{n}=9)$ no indicó con claridad sustancias que pudiesen ser clasificadas.

De los agroquímicos con efecto insecticida, los compuestos órgano fosforados se destacan como los más utilizados y entre ellos los

Tabla 1. Nivel de toxicidad de los agroquímicos utilizados en el medio y bajo sinú del departamento de Córdoba.

\begin{tabular}{lcc}
\hline $\begin{array}{c}\text { Nivel de } \\
\text { toxicidad }\end{array}$ & $\begin{array}{c}\mathbf{N}^{\circ} \mathbf{d e} \\
\text { fumigadores }\end{array}$ & \% \\
\hline Toxicidad I & 79 & 16,3 \\
Toxicidad II & 59 & 12,1 \\
Toxicidad III & 213 & 43,8 \\
Toxicidad IV & 126 & 25,9 \\
No clasifica & 9 & 1,9 \\
Total & 486 & 100 \\
\hline
\end{tabular}

Fuente: Información de la entrevista estructurada sobre condiciones de salud y trabajo. 
principios activos Metil parathión (63 reportes de uso), Clorpirifos (34 reportes), Malatión (8 reportes), dimetoato (7 reportes), profenofos (3 reportes); con relación a los carbamatos se encontró referencia a los ingredientes activos metomil (9) reportes, carbaril (6 reportes), thiodicarb (2 reportes); entre los piretroides el compuesto cipermetrina (20 reportes); entre los inhibidores de quitina el compuesto reportado fue el Lufenuron (26 reportes).

Entre los agroquímicos con efecto herbicida, utilizados en Córdoba, los compuestos Auxínicos o Fenoxi emergen como los más utilizados y entre este grupo el ingrediente activo glifosato (125 reportes), 2-4 D amina (21 reportes); entre los piridílicos el ingrediente activo paraquat (9 reportes). Los derivados picolínicos adicionados con auxínicos o fenoxí en especial el ingrediente activo Picloram+2,4D (6 reportes); del grupo de las triazinas el ingrediente activo utilizado es la Atrazina; en el grupo de las amidas el ingrediente activo referenciado fue el propanil (1 reporte); en el grupo de los ácidos alifáticos clorados el ingrediente activo refenciado fue el M.S.M.A (1 reporte).

El equipo investigador consideró de interés, clasificar la legalidad del plaguicida utilizado por el riesgo potencial para la salud pública, por las implicaciones para el medio ambiente y porque se sabe que estos compuestos están siendo ingresados y adquiridos como producto de contrabando en el departamento de Córdoba.

Las veinticuatro (24) sustancias químicas referidas por el trabajador rural como las más utilizadas, se clasificaron los compuestos desde la base de legalidad o ilegalidad de éstos para su uso en Colombia; se encontró que 20,8\% de dichos compuestos son de uso prohibido en el país. Se identificó que los jornaleros reportan la utilización en el último año de cinco compuestos prohibidos en Colombia (Tabla 2). Entre los productos prohibidos que se utilizan en Córdoba se encontraron Aldrín, Tiodan,
Lindano y DDT; es preocupante que estos plaguicidas se usen a pesar se ha comprobado el impacto negativo de éstos sobre el medio ambiente y su nivel de toxicidad sobre la salud de los seres humanos es reconocido en el territorio nacional.

Prácticas y conocimientos en el manejo de agroquímicos. Según el $89 \%$ de los participantes $(n=433)$, el transporte de los compuestos químicos desde el sitio de compra a la zona rural se realiza en vehículos de servicio público de manera conjunta con otros insumos e incluso el 56\%, lo realiza junto con los alimentos ( $n=272)$; aduciendo facilidades y menor costo en la movilización.

Entre los 362 operarios que se dedican de manera exclusiva a la fumigación se identifica que $73 \%$ almacena los químicos y las bombas mecánicas en lugares oscuros y con poca ventilación ( $n=264) ; 6,7 \%$ utiliza los gallineros $(n=24)$ y $13,1 \% \quad(n=74)$ en las habitaciones dentro de la vivienda, lo que incrementa la probabilidad de contacto innecesario y de riesgo de accidentes con agroquímicos.

El transporte desde el sitio de almacenamiento al de utilización se realiza $67 \%$ en bicicleta $(n=326), 12 \%$ en motocicleta $(n=58)$ y $15 \%$ $(n=73)$ en transporte "del patrón" al sitio de fumigación y al hombro $6 \%(n=29)$.

Es importante destacar que 24,3\% $\quad(n=118)$ de los fumigadores tiene la costumbre de leer la información que contiene la etiqueta del producto antes de aplicarlo, lo que puede atribuirse por una parte al alto porcentaje de analfabetismo entre los trabajadores agrícolas.

Se destaca que $14,4 \%(n=70)$ realiza habitualmente prácticas de manejo seguras y 10,2\% $(n=50)$ utiliza Elementos de Protección Personal (EPP) coherentes con el riesgo de exposición. Entre las prácticas de alto riesgo, en respuesta a este desconocimiento está que 13,1\% 
$(\mathrm{n}=64)$ de los sujetos de investigación reutiliza los recipientes de plaguicidas para transportar agua u otros líquidos para su consumo.

Con relación a la disposición final de los envases, $87 \%(n=423)$ de éstos son dispuestos en el campo, a las orillas de los cultivos y $13 \%$ $(n=63)$ en los basureros rurales sin que se realice ningún tipo de preparación de los envases para el descarte de los mismos, aumentando de forma inadvertida y permanente la contaminación progresiva de los suelos.
Conocimiento de los fumigadores con respecto a los riesgos para la salud. Para identificar el nivel y grado de apropiación de conocimientos sobre los riesgos personales y del medio ambiente relacionado con el uso de compuestos agroquímicos, se hicieron preguntas verificadoras acerca del conocimiento de los fumigadores y personal que trabaja con los plaguicidas sobre las sustancias químicas que usa en su cotidianidad laboral (Tabla 2).

Tabla 2. Conocimiento de los fumigadores sobre las sustancias químicas utilizadas en el trabajo

\begin{tabular}{lrc}
\hline \multicolumn{1}{c}{ Nivel de toxicidad } & $\begin{array}{c}\mathbf{N}^{\circ} \text { de } \\
\text { fumigadores }\end{array}$ & \% \\
\hline Reconoce que los agroquímicos son un riesgo para su salud & 267 & 68,1 \\
Detalla los diferentes riesgos a los cuales está expuesto por los agroquímicos & 50 & 10,2 \\
Describe las formas de prevención en el almacenamiento y transporte & 95 & 24,3 \\
Reconoce que el empaque informa la toxicidad y forma de descarte & 437 & 89,9 \\
Describe la forma o técnica segura de aplicación, almacenamiento, transporte y disposición & 70 & 14,4 \\
Final de sobrantes de mezcla, empaques y envases de agroquímicos & 95 & 24,3 \\
Detalla todas las vías de entrada de los tóxicos al organismo & & \\
\hline
\end{tabular}

Fuente: Información de la entrevista estructurada sobre condiciones de salud y trabajo.

Aunque siete de cada 10 fumigadores afirman que los agroquímicos representan riesgo para su salud, solo uno de cada diez está en condiciones de detallar los diferentes riesgos a los cuales está expuesto.

El 89,9\% de los fumigadores ( $n=437$ ) conoce que la etiqueta impresa en el envase del agroquími$\mathrm{CO}$, informa sobre la toxicidad de las sustancias y los mecanismos de descarte, sin embargo no saben explicar las razones por las que se deben utilizar dichas medidas; a falta de conocimiento utilizan una forma de lógica condicional para entender los riesgos del nivel de toxicidad, al respecto se pudo apreciar que los fumigadores informales entienden que el nivel de toxicidad I es el más leve o más inocuo y que el nivel IV es el más severo; siendo que la realidad toxicológica de los compuestos es inversa, condición que los predispone a mayor exposición con el consecuente riesgo para la salud de los fumigadores; por lo anterior es posible afirmar que los operarios fumigadores de los cultivos modales de Córdoba, desconocen en su mayoría los riesgos de los agroquímicos plaguicidas para su salud y para el medio ambiente.

Sin embargo, cerca del 25\% $(n=122)$ reconoce las posibles vías de entrada de los tóxicos al organismo, es capaz de describir los efectos nocivos y de enunciar al menos una forma de prevenirlos durante el transporte y almacenamiento, conocimiento tal vez aprendido por la experiencia propia y ajena.

En lo relativo a los riesgos para su salud personal, se vislumbra una problemática de salud pública que es potencialmente severa, si se considera que $89,8 \% \quad(n=436)$ de los fumigadores no utiliza los Elementos de Protección Personal (EPP) para reducir el contacto accidental con los agroquímicos plaguicidas durante su actividad laboral. En cuanto a los riesgos ambientales para la flora y la fauna, y sobre las técnicas de 
aplicación segura para el medio ambiente se pudo identificar que $85,6 \%(n=416)$ desconoce las formas y/o técnicas seguras de aplicación, formas de almacenamiento adecuadas, así como las técnicas apropiadas para el transporte y disposición final de sobrantes de mezcla, empaques y envases de agroquímicos.

En consecuencia, la situación encontrada entre los fumigadores de plaguicidas en los cultivos modales de Córdoba (Colombia), demuestra que utilizan de manera cotidiana es fragmentado e impreciso; no adquirido de manera formal, ni por parte de los agrónomos encargados de la asistencia técnica de los cultivos, sino que este conocimiento fragmentado es producto tanto de la tradición oral como de la experiencia personal y ajena.

Con relación a la participación del sexo femenino en este estudio, es significativo para esta discusión tener presente que, aunque nuestros resultados y otros estudios nacionales e internacionales se reportan escasas proporciones de mujeres desempeñándose como fumigadoras, estas cifras son relevantes por cuanto los efectos crónicos y retardados en la población femenina no sólo repercuten en el organismo de quienes están expuestas directamente al tóxico, sino que también implican a los embriones humanos expuestos y niños lactantes porque los tóxicos se transportan a través de la leche materna (Marc et al. 2002; OPS 2007; Cervantes 2010), las publicaciones citadas muestran el potencial letal del herbicida glifosato para las células humanas, se señala que "aun en dosis diluidas mil veces, los herbicidas cuyo principio activo es el glifosato estimulan la muerte de las células de embriones humanos, lo que podría provocar malformaciones, abortos, problemas hormonales, genitales o de reproducción (...), además de distintos tipos de cáncer", con dosis "hasta diez mil veces inferiores a las usadas en agricultura".
Aunque se ha demostrado que el glifosato a nivel de la fauna silvestre suele disminuir la tasa de supervivencia de diferentes artrópodos (Paganelli et al. 2010), este cambio altera la cadena trófica. Se destaca en la región escenario del estudio el predominio del uso del compuesto químico glifosato, el cual se prefiere para el control de gramíneas y otras especies de malezas, evidenciado en la frecuencia de 125 reportes de uso de herbicidas vs 63 reportes de insecticidas para control químico en cultivos modales también conocidos como cultivos industriales o tecnificados de maíz, arroz y algodón, así como también en cultivos de pan coger y en cultivos asociados (yuca// maíz//ñame). Coincidiendo con el estudio que reporta que el glifosato es utilizado con elevada frecuencia para el control de malezas (Rivera et al. 2007), a pesar de su potencial de afectación de algunas especies vegetales como son los arbustos perennes, el glifosato ha sido clasificado por la Unión Europea como "peligroso para el medio ambiente" y "tóxico para los organismos acuáticos". Situación preocupante para el entorno ambiental agrícola pues para mejorar la eficacia de los productos fitosanitarios el glifosato se combina con coadyuvantes y surfactantes, productos que agregados o incluidos en la formulación del herbicida, pueden resultar más tóxicos para el medio silvestre que el glifosato en sí mismo (Pengue 2003).

La Organización Mundial de la Salud ha clasificadoal Glifosato con toxicidad III (Williams et al. 2000), a pesar que en lo referente a la salud humana se ha demostrado que el glifosato tiene un efecto citotóxico y genotóxico en linfocitos humanos in vitro y expuestos durante 48 horas al tóxico (Díaz 2011). Considerando que resultados del presente estudio muestran que los fumigadores informales se exponen sin protección a los efectos de los agroquímicos en general, sus riesgos laborales deben ser un tema de alarma y preocupación para la salud pública (Williams et al. 2000). 
El desconocimiento de los fumigadores informales sobre los riesgos generados por los agroquímicos, puede darse al parecer a que políticamente no se le ha concedido la relevancia requerida, principalmente a que el sistema productivo y comercial de insumos propio del sector agrícola solo prevalecen las posibilidades del lucro por la comercialización, y lamentablemente la educación e información preventiva pasa a ser un asunto de segundo plano frente a los riesgos para la salud (Kaczewer 2010) y el ambiente, la cual se considera cubierto con las indicaciones de la etiqueta, sin embargo los fumigadores con bajo nivel de escolaridad no saben leer o interpretar el mensaje lo que justifica en gran medida el desconocimiento que existe sobre este tema por parte de los fumigadores. Visto desde otro ángulo no se entiende por qué a pesar de toda la documentación disponible sobre los efectos adversos para la salud laboral y para el medio ambiente en el entorno de las zonas agrícolas se desconoce esta información (OPS 2007; Cervantes 2010).

Es válido anotar que la División de Políticas Públicas de Chile (2014) ha demostrado que el escaso conocimiento de los usuarios sobre los riesgos de los agroquímicos, sumado a su amplia utilización, ha facilitado la aparición de intoxicaciones de tipo laboral o accidental, sin embargo esta secuencia de eventos parecería ser desconocida o intencionalmente ignorada en la región escenario de estudio, en la cual los fumigadores se exponen a los riesgos resultantes del contacto con agroquímicos (plaguicidas, herbicidas, fertilizantes), exponiéndose directa e indirectamente a factores de riesgo químico, peligro que se potencia ante la ausencia de asesoría técnica necesaria para la salud de los operarios, aspecto que debe ser una condición obligatoria en las buenas prácticas de producción agrícola.

Los hallazgos relativos a la disposición final de sobrantes y empaques del presente estudio coinciden con los resultados sobre las prácticas de descarte en las orillas de los cultivos y quema a campo abierto (Rivera et al. 2007); los autores citados encontraron evidencia de reutilización de los envases para almacenar y transportar líquidos para consumo humano. Los mismos autores reportan otras prácticas evidentemente nocivas como disposición final de sobrantes y envases en arroyos, ríos, canales de drenaje, ciénagas y demás acuíferos, ocasionando daños ecológicos incalculables e ignorando el efecto tóxico a nivel humano y ambiental.

Es relevante recordar que el glifosato tiene una alta capacidad de mantener su estabilidad y, debido al potencial de difusión, puede contaminar las aguas superficiales y subterráneas, más aún si se considera que la toxicidad del glifosato en agua se incrementa con las temperaturas más altas, condición común en ambientes de trópico bajo como es la región del Sinú medio y bajo que sirvió de escenario al estudio. Los peligros ambientales a los que se hace referencia son que el glifosato es levemente tóxico para algunos anfibios y peces, pero la distribución de ambas poblaciones también puede verse afectada al destruirse las fuentes de alimento de su hábitat (Pengue 2003).

Un interesante hallazgo del presente estudio, es la demostración del uso habitual de algunos compuestos agroquímicos prohibidos en Colombia, lo cual es indicativo de otra forma de ejercicio de prácticas nocivas para la sostenibilidad ambiental (ICA 2004; Badii et al. 2007) y humana (Díaz. 2011) por motivos que pueden estar relacionados con diversos factores como el económico, debido a los bajos precios de estos productos de contrabando, el apego cultural a ciertas sustancias plaguicidas, la disponibilidad de los mismos en el mercado o el desconocimiento de las causas por las cuales éstos están prohibidos incluyendo los riesgos potenciales para la salud de las generaciones presentes y futuras. Incluso es posible que los patronos o 
contratistas los prefieran por la alta efectividad, por la larga duración de los efectos de este tipo de agroquímicos ignorando intencionalmente ó prefiriendo desconocer la evidencia científica de los impactos de dichos insumos agrícolas sobre la salud humana y del ambiente.

En este mismo orden, la Organización Panamericana de la Salud (OPS 2007), corrobora que en muchos ámbitos, como en la región del Bajo y Medio Sinú de Córdoba (Colombia) no se tiene ningún tipo de control sobre el manejo de envases de agroquímicos y la gestión de los mismos, trayendo como consecuencia la contaminación irreversible del ambiente. El mismo autor señala que la disposición final de estos recipientes no tiene un manejo adecuado: $35 \%$ los arroja a las basuras, $23,7 \%$ los queman a cielo abierto ocasionando una emisión incontrolada de dioxinas y furanos si los envases son de plásticos clorados, 6,9\% los entierra sin tratamiento, $13,3 \%$ los acumula en el campo o en galpones y $4 \%$ los reutiliza. En Colombia, a la fecha aunque tiene una orientación de la autoridad ambiental sobre el destino final de estos residuos peligrosos, ésta no se hace realidad en las zonas en las cuales se utilizan estos insumos químicos agrícolas.

Bajo este panorama se pueden visualizar cuan severos son los riesgos personales a los que se exponen los operarios fumigadores informales, máxime cuando realizan cotidianamente su actividad laboral sin la apropiada protección respiratoria $(93 \%)$, ni de piel $(87 \%)$, cifras coincidentes con los hallazgos que señalan que del total de productores $5 \%$ se protege de alguna manera (Montoya et al. 2007), la mayoría de ellos debido al desconocimiento de las implicaciones de los químicos para su salud (cáncer, intoxicación aguda, intoxicación crónica, síndromes neurotóxicos) se expone de manera continua a los efectos negativos de los agroquímicos plaguicidas sobre la base de una falsa sensación de confianza, tanto en las técnicas que utiliza como en las propias sustancias que son esparcidas en el acto de la fumigación, sin saber que además se induce a la contaminación progresiva o continuada por compuestos químicos (Ortiz et al. 2011).

Estos compuestos químicos se esparcen de manera habitual con bomba manual de espalda, las cuales por lo general están en mal estado mecánico (Montoya et al. 2007), lo que induce a goteo continuo de dosis variables y concentradas del químico agrícola en un punto que afecta de manera progresiva la condición de salud del operario y también induce a contaminación progresiva del medio ambiente e incluso de la fertilidad del suelo al reducir la microflora y microfauna requerida para los ciclos del nitrógeno, situación que es agravada por la dispersión de agroquímicos de uso ilegal que han sido prohibidos por su alta persistencia (Barba-Ho y Becerra 2011).

\section{CONCLUSIONES}

Las prácticas agrícolas observadas entre los fumigadores del Medio y Bajo Sinú, relacionadas con agroquímicos, se realizan en contextos de desconocimiento sobre riesgos químicos, dentro de estas se mencionan manejo seguro de plaguicidas, formas de eliminación e importancia del uso de EPP. Así mismo, se efectúan en condiciones de desprotección laboral con potenciales de daño a la salud humana y ambiental, situación que se magnifica con la utilización persistente de compuestos no permitidos en Colombia.

La situación arraigada de exposición al riesgo sin protección laboral, al parecer, ha sido generada entre muchos factores por trabajar en el sector informal de la economía, alto grado de analfabetismo, desconocimiento sobre manejo de riesgo químico y prevención del mismo, experiencia acumulada en la exposición arbitraria que se manifiesta en familiarización con el riesgo. 
La condición de fragilidad, insalubridad y desprotección en la que laboran estos fumigadores, produce consecuencias negativas a nivel individual, familiar y social, las cuales deben ser intervenidas desde la Política Agraria, el ámbito de la Salud Pública, Salud laboral y Epidemiología, de forma tal que la reformulación de las Políticas Públicas garantice la Seguridad y Salud en el Trabajo, al cual tienen derecho constitucional, ético, moral y legal de estos colectivos humanos.

\section{REFERENCIAS}

Badii. M, Garza, R., Garza, V. y Landeros, J. 2005. Los indicadores biológicos en la evaluación de la contaminación por agroquímicos en Ecosistemas acuáticos y asociados. CUICYT, 6, 4-20

Badii, M. y Landeros, J. 2007. Plaguicidas que afectan a la salud humana y la sustentabilidad. CULCyT, 4(19), 21.

Barba-Ho, yBecerra, D.2011. Biodegradabilidad y toxicidad de herbicidas utilizados en el cultivo de caña de azúcar. En: Ingeniería de Recursos Naturales y del Ambiente.

Barreto., G. Pérez De Corcho, O. 1989. Efecto de pesticidas sobre la microflora del suelo. Revista de Producción Animal. CU. Vol.5. Num.1

Fedesarrollo-Iquartil. 2013. Costos de Producción de Doce Productos Agropecuarios. http://www.fedesarrollo.org.co/wp-content/uploads/2013/02/IF-Costos-agropecuarios_FINAL.pdf. Pág. 181

Cervantes, R. 2010. Plaguicidas en Bolivia: sus implicaciones en la salud, agricultura y medio ambiente. Revista Virtual REDESMA. Vol. 4(1) Pág. 6, (1-12). Disponible en: http://www.cebem.org/cmsfiles/articulos/REDESMA_09_art02.pdf
Chile. División de políticas públicas saludables y promoción. Departamento de Salud Ocupacional. 2014. Protocolo de vigilancia epidemiológica de trabajadores expuestos a plaguicidas. 2014. Pág. 4 Disponible en http://web.minsal.cl/sites/ default/files/Protocolo_de_Vigilancia_ Trabajadores_Expuestos_Plaguicidas.pdf

Díaz, A. 2011. Efecto citotóxico y genotóxico del glifosato en linfocitos humanos. Universidad Veracruzana. México. Facultad de Biología. Tesis para optar el título de Licenciado en Biología.

Duran-Nah, J. y Colli-Quintal, J. 2000. Intoxicación aguda por plaguicidas. Salud pública Méx [online], vol.42, n.1, pp. 48-52. ISSN 0036-3634.

Federación de Aseguradores Colombianos. FASECOLDA. 2013. Estadísticas del ramoenfermedades profesionales 2000-2011 [En línea]. [Consulta 17 febrero 2014] disponible en: http://www.fasecolda. com/index.php/ramos/riesgos-laborales/ estadisticas-del-ramo

Instituto Colombiano Agropecuario (ICA), Subgerencia de Producción Agrícola, División de insumos agrícolas. Colombia. 2004. Resolución 001973 del 15 de septiembre de 2004 del ICA. 2004. [En línea]. [Consulta 10 noviembre de 2015] disponible en: www.ica.gov.co/getdoc/ b2e5ff99-bd80-45e8.../PLAGUICIDASPROHIBIDOS.aspx

Kaczewer, J. 2009. Subestimación del impacto de la exposición a agrotóxicos sobre la salud humana. En:http://vozentrerriana. blogspot.com/2009/08/agroquimicospatente-para-matar.html. Publicado septiembre 15 de 2009.

Marc, J., Mulner-Lorillon, O., Boulben, S., Hureau, D., Durand, G. and Bellé R. 2002. Pesticide Roundup provokes cell division dysfunction at the level of CDK1/ cyclin B activation. Chem Res Toxicol; 15 (3): 326-31. 
Ministerio de Ambiente, Vivienda y Desarrollo Territorial Dirección de Desarrollo Sectorial Sostenible. 2008. Uso aparente de plaguicidas en Colombia durante los años 2004 - 2007. (s.f). Pág. 13-16

Montoya, R., Pérez, Y. y Vitoria, R. 2007. Métodos y/o prácticas de aplicación de agroquímicos empleadas por los productores agrícolas en el Departamento de Córdoba. En: Memorias XXXVII Congreso Anual Comalfi. Pág. 38

Organización Panamericana de la Salud, España. Ministerio de Salud. Presidencia de la Nación y Secretaria de Ambiente y Desarrollo Sustentable. 2007. La problemática de los agroquímicos y sus envases, su incidencia en la salud de los trabajadores, la población expuesta y el ambiente. Provincia de Catamarca. Parte 3. Estudio colaborativo multicéntrico. En: https:// www.estrucplan.com.ar/Producciones/ imprimir.asp? IdEntrega $=2446$

Ortiz, R., Villadiego, J. y Cardona, C. 2011. Valoración de los impactos ambientales totales generados por el uso de plaguicidas en actividades ganaderas en el municipio de Pamplona -Norte de Santander - Colombia. En: Revista de Didáctica Ambiental Año 7 No 10. Diciembre. Pág. 70

Paganelli, A., Gnazzo, V., Acosta, H., López, S. y Carrasco, A. 2010. Herbicidas a base de glifosato producen efectos teratogénicos en vertebrados al perjudicar la señalización del ácido retinoico. En: Chem Res Toxicol .18; 23 (10): 15861595. doi: 10.1021 / tx1001749. Epub 201009 de agosto
Pengue, W. 2003. El glifosato y la dominación del ambiente. En: Biodiversidad. Pág. 5-6

Ramírez, J. y Lacasaña, M. 2001. Plaguicidas: clasificación, uso, toxicología y medición de la exposición. En: ArchPrev Riesgos Labor; 4(2):67-75

Rivera, C, Pérez, Y. y Vitoria, R. 2007. Agroquímicos con mayor incidencia de uso para el control de arvenses en los principales cultivos del Departamento de Córdoba. En: Memorias XXXVII Congreso Anual Comalfi. Pág. 2538

SIVIGILA Grupo Factores de Riesgo Ambiental, Subdirección de Vigilancia en Salud Pública, INS. 2014. En: www. minproteccionsocial.gov.co/.../Boletin_ epidemiologico_Seman.

Guzmán, C., Villa, H., Calderón A. 2015. Análisis epidemiológico y clínico de intoxicaciones agudas atendidas en Montería, Colombia. En: Rev. Méd. Risaralda 2015; 21 (1): 17-21.

Williams, G., Kroes, R. and Munro, C. 2000. Safety evaluation and risk assessment of the herbicide Roundup and its active ingredient, glyphosate, for humans. En: Regulatory Toxicology and Pharmacology, 31 (2): págs. 117-165, PMID 10854122. 